\title{
Organes supplémentaires : machines et moyens de communication dans les derniers romans de Claude Simon
}

Supplementary Organs: Media and Machinery in the Late Novels of Claude Simon

\section{Wolfram Nitsch}

\section{(2) OpenEdition Journals}

\section{Édition électronique}

URL : https://journals.openedition.org/ccs/2373

DOI : $10.4000 /$ ccs. 2373

ISSN : 2558-782X

\section{Éditeur :}

Presses universitaires de Rennes, Association des lecteurs de Claude Simon

\section{Édition imprimée}

Date de publication : 30 septembre 2019

Pagination : 195-211

ISBN : 978-2-7535-7795-4

ISSN : $1774-9425$

\section{Référence électronique}

Wolfram Nitsch, «Organes supplémentaires : machines et moyens de communication dans les derniers romans de Claude Simon », Cahiers Claude Simon [En ligne], 14 | 2019, mis en ligne le 30 septembre 2020, consulté le 25 avril 2022. URL : http://journals.openedition.org/ccs/2373; DOI : https://doi.org/10.4000/ccs.2373 


\title{
ORGANES SUPPLÉMENTAIRES : MACHINES ET MOYENS DE COMMUNICATION DANS LES DERNIERS ROMANS DE CLAUDE SIMON
}

\author{
Wolfram NITSCH \\ Université de Cologne
}

La technologie apparaît dans l'œuvre de Claude Simon comme une thématique et un champ métaphorique majeurs. Dans Le Vent, l'un de ses premiers romans, Montès, le personnage principal, ne se sépare jamais de son appareil-photo, observant à travers l'objectif comme il le ferait à travers la vitre d' " une auto, un tramway, un train, un véhicule en marche" $(V, \mathrm{p} .155)$. De même, dans Le Jardin des Plantes (1997), le protagoniste et narrateur S. est obsédé par les machines et les moyens de communication. Au sein de l'exploitation littéraire de ce champ par Simon, on peut distinguer trois perspectives distinctes, mais qui souvent se recoupent. La première : plusieurs références relèvent d'une critique polémique de la technologie considérée comme totalement extérieure et de ce fait dangereuse pour l'homme. Cette critique fait écho au pessimisme culturel exprimé par Spengler et ses précurseurs romantiques, qui mettaient en garde contre la soumission de l'homme à la machinerie industrielle, en affirmant que, loin d'accroître sa puissance, elle ne faisait que l'usurper ${ }^{1}$. C'est ainsi que les membres de la société dans laquelle vit Montès

1. O. Spengler, "La machine ", dans Le Déclin de l'Occident, Esquisse d'une morphologie de l'histoire universelle [1922], trad. M. Tazerout, Gallimard, coll. «Bibliothèque des idées », 1948, t. 2, p. 458-467. On trouve une critique romantique influente de la machinerie industrielle chez Michelet, l'un des auteurs favoris de Simon au xIx siècle. Voir P. Viallaneix, « Michelet, machines, machinisme », Romantisme, n 23, 1979, p. 3-15. 
appartiennent à une " espèce nouvelle " décadente, une espèce incapable de survivre sans les artefacts techniques :

sorte de ver blanc et mou de fabrication récente, issu [...] selon toute apparence du coït entre l'automobile et le radiateur de chauffage central, totalement inapte à se mouvoir autrement qu'à l'aide d'un moteur, à se distraire qu'en technicolor et à se concevoir qu'en monnaie-papier. $(V$, p. 80$)$

Quoique nécessaires, ces artefacts menacent toujours de se retourner contre leurs inventeurs et leurs utilisateurs; en particulier en temps de guerre, ils prennent leur revanche, révélant "l'inflexible perfidie des choses créées ou asservies par l'homme " $(R F$, p. 232). En second lieu, pourtant, un certain nombre de passages chez Simon portent un regard plutôt anthropologique sur la technologie pour y voir une extension indispensable à l'homme. Suivant certaines tendances importantes de l'anthropologie moderne, ils soulignent le rôle de complément que joue le progrès technique en affirmant que toute expérience humaine du monde est toujours déjà guidée par elles ${ }^{2}$. L'appareilphoto de Montès, par exemple, est comparé à un "troisième œil, un organe supplémentaire » $(V$, p. 15) qui, en tant que tel, offre des perspectives inédites. Paradoxalement, ce rôle crucial de l'instrument se manifeste surtout quand il ne marche pas parfaitement, quand les composantes du dispositif bénéficient de trop de jeu, car c'est alors seulement, comme l'observe Simon, en citant Heidegger, qu'il attire une attention qu'on ne lui accorde jamais par ailleurs : "C'est dans ce découvrement de l'inutilisable que soudain l'outil simpose à l'attention" (BP, p. 685, italiques de Simon). Enfin, la technologie apparaît souvent dans une perspective esthétique. Il n'est pas rare de voir des romans de Simon faire référence à des innovations techniques qui stimulent ou illustrent l'écriture. À la suite de Proust auquel il voue une grande admiration, et de ses successeurs Beckett ou Leiris, il utilise la technique et les moyens de communication dans une logique productive en les présentant comme des moteurs ou des modèles puissants de l'imagination littéraire, et

2. Cette position anti-rousseauiste caractérise également l'anthropologie philosophique de Gehlen, la théorie des médias de McLuhan et l'anthropologie culturelle de Leroi-Gourhan. Voir A. Gehlen, "L'homme et la technique ", dans Anthropologie et psychologie sociale [1957], trad. J.-L. Bandet, PUF, coll. «Philosophie d'aujourd'hui », 1990, p. 173-191; M. McLuhan, Pour comprendre les médias [1964], Le Seuil, coll. " Points ", 1977, en particulier le chap. 4; A. Leroi-Gourhan, Le Geste et la parole, vol. 1, Albin Michel, 1964. La dernière étude constitue un point de référence important pour la théorie derridienne de l'écriture, centrée sur le concept de «supplément ». Voir J. Derrida, De la grammatologie, Minuit, 1967, p. 207-234. 
parfois même de l'initiation littéraire ${ }^{3}$, même si cela ne s'accomplit qu'au moyen d'artifices poétiques, en particulier la métaphore. Vue sous cet angle, l'écriture elle-même ressemble à " un organe supplémentaire » $(R F, \mathrm{p} .214$, 362). D'un côté, elle semble n'être que l'un de ces artefacts utilitaires, très proche en cela d'objets techniques culturellement moins prestigieux qu'elle. D'un autre côté, elle joue le rôle d'un super-médium qui ouvre des perspectives esthétiques qui dépassent de loin l'usage conventionnel de ces objets. Cette triple représentation par Simon de la technologie comme extériorité inorganique et dangereuse, comme extension quasi organique indispensable, et comme stimulus esthétique potentiel, est particulièrement saillante dans les trois derniers romans, où la technique et les moyens de communication occupent une place centrale. Pour cette raison, nous concentrerons notre réflexion sur ces romans, en nous appuyant principalement sur les exemples du magnétophone dans Le Jardin des Plantes, du train dans L'Acacia et du cinéma dans Les Géorgiques.

\section{LE MAGNÉTOPHONE DANS LE JARDIN DES PLANTES}

Parmi les instruments techniques que les personnages simoniens observent ou utilisent, les moyens de communication sonores apparaissent assez tardivement. Ils sont quasiment absents de leur univers jusqu'aux Corps conducteurs, où le personnage central écoute d'interminables discours déformés par un amplificateur et utilise à plusieurs reprises un téléphone, tentant vainement de joindre son ancienne maîtresse ${ }^{4}$ (CC, p. 472-485). Ce n'est qu'avec Le Jardin des Plantes que l'on retrouve une confrontation aussi intense avec un média audiophonique. Dans ce roman, le narrateur quasi-autobiographique (il s'appelle parfois lui-même S.) se remémore à plusieurs reprises les fragments d'une longue interview accordée dans les années 1990 à un jeune journaliste qui l'enregistre sur magnétophone. Au début de l'entretien, il déclare ouvertement sa profonde aversion pour l'outil ("Je n'aime

3. Sur l'usage esthétique de la technologie chez Proust, voir p. ex. M. Schneider, Die erkaltete Herzensschrift. Der autobiographische Text im 20. Jahrhundert, Munich, Hanser, 1986, p. 93-103, et mon étude "Fantasmes d'essence. Les automobiles de Proust à travers l'histoire du texte ", dans R. Warning et J. Milly (dir.), Marcel Proust. Écrire sans fin, CNRS, 1996, p. 125-141.

4. Avant Les Corps conducteurs, le microphone apparait seulement dans Histoire (p. 364-366), et le téléphone seulement dans la bande dessinée évoquée dans La Bataille de Pharsale (p. 605-608). Pour un aperçu rapide des références de Simon au téléphone inspirées par celles de Proust dans la Recherche, voir F. Schuerewegen, "Orphée au téléphone. Appel et interpellation chez Claude Simon ", Poétique, $\mathrm{n}^{\circ}$ 76, 1988, p. 451-461. 
pas beaucoup cet instrument ", p. 957), et le développement qui suit évoque le magnétophone de façon très négative en soulignant les perturbations et les déformations qu'implique son utilisation. Tout d'abord, l'enregistrement est sous la menace permanente d'une interruption liée aux aléas techniques. À plusieurs reprises, le récit ému par S. de ses souvenirs de guerre est brutalement interrompu parce que l'intervieweur doit vérifier son appareil (p. 959, 970) ou changer la bande (p. 972, 1103). Ensuite, la voix enregistrée du locuteur est si différente de la voix intérieure qu'il est habitué à entendre, qu'il ne parvient pas à la reconnaître pour sienne. En réécoutant une partie de l'interview, S. entend une voix étrange qui semble émaner de l'appareil luimême, " une voix métallique, timbrée, qui n’est pas la mienne ou du moins celle que j'entends quand je parle» (p. 959). Au bout de quelques minutes, il commence à souffrir d'une sorte d'aliénation auditive qui ressemble à celle du Krapp de Beckett quand il entend un vieil enregistrement de sa propre voix qui lui semble celle d'un idiot inconnu ${ }^{5}$.

Toutefois, à mesure que l'entretien se poursuit, $S$. commence à sentir que son acuité auditive est rendue plus affûtée par la présence du magnétophone. Plus la conversation se prolonge, plus il se fait attentif aux sons qui l'environnent. Cela tient au fait que l'appareil enregistreur ne fait pas la différence entre le discours et le bruit qui l'accompagne. Peu après le début de l'interview, le journaliste interrompt subitement l'enregistrement parce que la voix de son interlocuteur est recouverte par le vrombissement d'un moteur (p. 970). Même si ces bruits de fond très sonores cessent rapidement, S. est désormais très attentif à tout ce qui peut s'entendre en plus de ses propres paroles, y compris les silences qui les séparent. Vers la fin de l'interview, il semble être obsédé par un bruit léger qui fait écho à celui du moteur au début, le " ténu et tenace bourdonnement (ou plutôt chuintement) d'un moteur au point mort, tournant à vide mais obstiné " (p. 1112). Ce bruit lui apparaît comme une manifestation acoustique menaçante du monde extérieur, un "bruit de fond "suggérant terreur et violence (p. 1120). En cela, la rumeur grondante de la ville lui rappelle l'expérience traumatisante de la guerre qu'il tente désespérément de communiquer au journaliste. Elle rappelle non seulement le chaos assourdissant de tous les engins de guerre modernes, mais aussi

5. S. Beckett, La Dernière bande, Minuit, 1959, p. 17 et 27. Avant même la pièce de Beckett, toutefois, la voix de l'" idiot " Montès se caractérise par une "voix de phonographe " $(V$, p. 14). Sur la tendance générale de Simon à présenter la voix comme quelque chose d'absent ou d'extérieur au locuteur, voir C. Britton, "Voice, absence and presence in the novels of Claude Simon ", French Studies, vol. 36, 1982, p. 445-454. 
le «fracas silencieux" (CC, p. 468) qui revient souvent dans les romans de Simon, ce bruit léger du temps lui-même qui devient audible entre ou après les batailles ${ }^{6}$. Ainsi, la conversation enregistrée donne lieu à une expérience auditive de la temporalité. Chaque fois que le discours de $S$. est perturbé ou interrompu, son attention est attirée vers ce que Blanchot appelle "le murmure ", un bruit impersonnel et étrange propre à tout discours mais en principe recouvert par lui ${ }^{7}$. Sauf que pour Simon, ce détournement de l'attention n'est pas seulement l'occasion, comme chez Blanchot, d'un usage poétique du langage qui place ce murmure au premier plan. C'est plutôt un phénomène acoustique qui permet de révéler la menace d'interruption constante que les bruits contingents font peser sur la communication verbale ${ }^{8}$. De même que, dans Les Corps conducteurs, le "silencieux fracas " est révélé par le téléphone (p. 472), le " ténu et tenace bourdonnement » dans Le Jardin des Plantes n'aurait pas été audible sans l'enregistrement.

L'avant-dernier roman de Simon relie donc perception imaginaire du temps et utilisation d'un média technique. Le rôle de la littérature comme médiateur nécessaire ne s'en trouve pas réduit pour autant. Cela devient évident à la fin, quand $S$. travaille au scénario d'une adaptation cinématographique de l'un de ses romans ${ }^{9}$. Une longue séquence évoque ce même bureau de l'écrivain où s'est déroulée l'interview. Et comme précédemment, le scénario insiste sur le vacarme du trafic : " au risque même de lasser le spectateur, le grondement des voitures est très violent " (p. 1174). À la fin, le bruit harassant est remplacé par celui de la machine à écrire, un bruit qui est lui-même peu à peu remplacé par celui des sabots des chevaux qui sert de transition vers une scène tirée des expériences de $S$. à la guerre. La bande-son très élaborée du projet cinématographique de $S$. en apporte la preuve : la perception des bruits

6. C'est particulièrement le cas dans La Route des Flandres, où entre l'extermination de l'escadron de de Reixach et la mort violente de ce dernier, le « silence au deuxième degré " que constitue le martèlement des chevaux du régiment révèle "le cheminement même du temps " (p. 212), le "destructeur travail du temps" (p. 412).

7. M. Blanchot, « Mort du dernier écrivain », Le Livre à venir [1959], Gallimard, coll. " Folio essais », 1986, p. 296-302. Sur la familiarité de Simon avec Blanchot, qu’il a rencontré à l'occasion du "Manifeste des 121 » en 1960, voir son interview avec Bernard-Henri Lévy dans Les Aventures de la liberté, Grasset, 1991, p. 12-21.

8. Pour une analyse brillante, quoique dans une certaine mesure déterministe, de l'influence de cette expérience des outils de la communication sur la littérature moderne, voir F. Kittler, Grammophon Film Typewriter, Berlin, Brinkmann et Bose, 1986, p. 35-173.

9. En 1977, Simon a écrit lui-même le scénario d'une adaptation de La Route des Flandres qui n'a jamais été tournée. Voir sa lettre à Jean Dubuffet dans leur Correspondance 1970-1984, L'Échoppe, 1994, p. 23. 
qui passe par l'enregistrement ne peut se transformer en expérience du temps que par le pouvoir médiateur de l'écriture. En un sens, cette toute-puissance a déjà été démontrée lors de la scène de l'interview. En effet, le bruit troublant qui émane de l'extérieur n'est pas seulement perçu, il est en même temps produit par une chaîne de signifiants : " ténu et tenace bourdonnement (ou plutôt chuintement) d'un moteur au point mort, tournant à vide mais obstiné ». Ici, le bourdonnement incessant est suggéré par l'expression onomatopéique et la répétition phonétique. Les termes allitératifs " ténu ", " tenace " et " tournant ", associés à " plutôt ", " moteur " et " obstiné ", suggèrent un son mécanique, le même "Ttt » utilisé auparavant pour exprimer le " déclic " du magnétophone (p. 972). La rime des termes " bourdonnement ", " chuintement " et «tournant" imite un monotone ostinato. C'est ainsi que la leçon acoustique du moyen de communication se voit transformée en expression poétique. Sur ce point, Simon emboîte le pas à Leiris, auquel il fait allusion, quoique d'une façon assez polémique, à la fin du roman ${ }^{10}$. Dans un chapitre de son autobiographie La Règle du jeu, Leiris explique comment l'utilisation du phonographe l'a prématurément conduit à une expérience acoustique de la mort, expérience qu'il évoque au moyen d'un jeu de mot suggestif autour du nom mythique de Perséphone ${ }^{11}$. Dans Le Jardin des Plantes, les moyens de communication audio sont utilisés et intégrés sur un mode similaire : par l'onomatopée et d'autres moyens poétiques, $\mathrm{S}$. (re-)crée ce bruit du temps et de la violence qu'il a appris à percevoir avec l'aide de ce magnétophone tant détesté.

\section{LE TRAIN DANS L'ACACIA}

Le premier exemple l'a montré, le bruit omniprésent que révèlent les instruments audiophoniques est, chez Simon, étroitement lié à celui des machines. Deux types de machines attirent principalement l'attention de

10. Il est cet homme aux airs de dandy, " maître de maison au crâne non pas chauve mais tondu comme celui d'un bagnard, au visage torturé de bagnard, tordu ou plutôt convulsé ", qui fait donner chez lui la première d'une représentation théâtrale peu avant la libération de Paris (p. 1151). La pièce à laquelle il est fait allusion est Le Désir attrapé par la queue de Picasso, jouée en juin 1944, en présence - et dans certains cas, avec leur participation - d'un groupe d'intellectuels parisiens, parmi lesquels Sartre, Camus, Lacan et - dans le rôle de l'obscur " cousin de province " (p. 1150) - le jeune Simon lui-même. Pour preuve de son admiration - rarement admise - pour Leiris l'écrivain et La Règle du jeu, voir l'entretien de Simon avec Madeleine Chapsal dans Quinze écrivains, Julliard, 1963, p. 169.

11. M. Leiris, Biffures, Gallimard, 1948, p. 86-101. Le nom de Perséphone, déesse du monde souterrain, est associé à l'idée d'une " percée " menaçante, ainsi qu'au " phonographe " ou au " graphophone " dont les craquements produisent des fortes perturbations auditives. 
ses personnages et narrateurs. L'un est agricole, par exemple le vestige de tracteur, décrit comme une "machine " paradigmatique dans La Bataille de Pharsale (p. 658-662); l'autre occupe une place plus importante : il s'agit du train, présent dans presque tous les romans et presque toujours associé à la guerre. Chez Simon, les trains mènent directement aux champs de bataille ou aux camps de prisonniers, ou encore, à tout le moins, aux souvenirs qui y sont associés ${ }^{12}$. L'Acacia offre des exemples de ces deux types de voyage. Durant le trajet que le protagoniste effectue en train pour rejoindre le front, d'autres périples du même ordre lui viennent à l'esprit : tandis qu'il effectue le long voyage qui le mène du Midi vers les Flandres à la suite de la mobilisation générale de 1939, il se souvient des voyages à Barcelone et à Moscou en 1937, et il anticipe son convoyage vers un camp saxon en 1940. À cette occasion, le train est constamment caractérisé comme un véhicule pernicieux qui déclenche ou commet la violence, un assemblage menaçant ( $A$, p. 1109) qui inspire un sentiment d' "instinctive horreur" (p. 1101). Il menace tout d'abord de blesser le personnage en secouant son corps et son esprit $^{13}$. Le "bruyant fracas " des roues et les " chocs réguliers " causés par la jointure des rails semblent provoquer un tremblement de terre artificiel (p. 1112), un " cataclysme " d'origine humaine qui détruirait son inventeur et ses utilisateurs (p. 1102). Qui plus est, le train favorise la formation d'amples foules humaines qui absorbent l'individu. Quand le convoi militaire quitte la gare, les personnes qui se trouvent sur le quai sont pressées les unes contre les autres au point de former un " conglomérat humain », une " marée humaine " anonyme (p. 1103). Le même phénomène se produit ensuite à l'intérieur du train. Dans le wagon de marchandises qui se dirige vers le front, les soldats se voient transformés en horde sauvage (p. 1154); dans le wagon à bestiaux qui roule vers le camp, les prisonniers deviennent un "informe et vague agrégat ", plus proche du troupeau de bêtes que d'un groupe d'hommes (p. 1207). Et surtout, le train apparaît comme l'élément central de la machine de guerre continentale. Cette association technique de locomotives puissantes et de grandes lignes ferroviaires fournit une image impressionnante de la méca-

12. Voir par exemple le train qui mène à la guerre civile espagnole dans Le Palace (p. 437-470), le convoi militaire vers les Flandres dans Les Géorgiques (p. 693-698), ou le train de prisonniers à destination de la Saxe évoqué à travers La Route des Flandres. Voir encore le voyage en train après la guerre dans La Bataille de Pharsale, qui suscite constamment des images de violence (p. 662-680).

13. Les deux dangers sont évoqués avec concision dans Les Géorgiques, où les passagers du train subissent un " choc brutal qui se répercute douloureusement depuis les talons jusque dans la boîte crânienne " ( $G$, p. 695). Pour une approche historique de la réflexion sur ces dangers, voir W. Schivelbusch, Histoire des voyages en train [1977], trad. J.-F. Boutout, Le Promeneur, 1990, chap. 9. 
nique de guerre moderne ${ }^{14}$. C'est ainsi que le convoi militaire à destination des Flandres est décrit comme

un de ces trains qui tous ensemble, au même moment, grondaient sur des ponts, s'engouffraient dans des tunnels, franchissaient des fleuves, sifflaient lugubrement, haletaient à travers les plaines d'un continent couturé de cicatrices, cousu et recousu tant bien que mal comme on recoud tant bien que mal le ventre ou le poitrail des chevaux déchirés par les cornes du taureau pour les lui présenter à nouveau (p. 1127)

Le train qui transporte le protagoniste n'est que l'un de ces innombrables véhicules du même genre, qui roulent tous en même temps et forment ainsi une énorme machine, "quelque invisible et impitoyable machine» (p. 1248); omniprésents, les rails évoquent les cicatrices d'une Europe que le taureau de la mythologie n'aurait plus violée, mais lacérée. Par ce genre d'images, le roman de Simon réactive le mythe pessimiste du XIX ${ }^{\mathrm{e}}$ siècle qui voit dans la machine à vapeur un monstre destructeur, que Zola met en scène avec la plus grande vigueur dans les Rougon-Macquart ${ }^{15}$. Ce n'est pas par hasard que tous ces trains sont tirés par de vieilles locomotives, qui déversent bruyamment leur vapeur d'un théâtre militaire à l'autre.

Pour le voyageur de L'Acacia cependant, le train ne constitue pas seulement une machine de mort impersonnelle. Pendant son long voyage vers le front, il l'utilise aussi comme un moyen personnel pour voyager par les sens et l'imagination, qui offre une expérience particulière du temps et de l'espace ${ }^{16}$. Cela lui permet d'abord d'entendre des choses qui, autrement, lui auraient échappé. Être étendu à même le sol du wagon, la tête non loin des roues et des rails, lui permet d'imaginer l'" inquiétant et inaudible tonnerre " de tous les trains qui traversent le continent, quelque chose de très semblable à cet étrange bruit révélé par le magnétophone (p. 1170). En outre, par l'accélération du rythme d'apparition et de disparition d'objets qui passent comme un éclair, le train suscite une dynamique, et parfois même une vision fantasmagorique

14. Voir W. Schivelbusch, op. cit., chap. 2, et D. Pick, War Machine: The Rationalization of Slaughter in the Modern Age, New Haven, CT, Yale University Press, 1993, p. 35-36 et 101-110. La métaphore de la guerre industrialisée en "énorme machine » ferroviaire apparaît dès Les Géorgiques (p. 729-731).

15. Pour une reconstruction détaillée de ce "mythe littéraire de la machine ", voir J. Noiray, Le Romancier et la machine. Limage de la machine dans le roman français (1850-1900), Corti, 1981-82, vol. 1, p. 424-447, et vol. 2, p. 391-397.

16. Voir la note de Maurice Merleau-Ponty sur le «temps de la nuit ferroviaire, des wagons " dans les romans de Simon ("Notes de cours "Sur Claude Simon” " [1961], Genesis, vol. 6, 1994, p. 144). Voir également J. Duffy, "Claude Simon, Merleau-Ponty and perception ", French Studies, vol. 46, n ${ }^{\circ} 1$, 1992, p. 33-52. 
du monde parcouru ${ }^{17}$. L'image de la foule sur le quai par exemple se modifie rapidement quand le train démarre, les visages devenant des "taches ", puis des points, puis " rien qu'un indistinct et sombre agrégat » (p. 1106); ce qui était auparavant présenté comme l'effet d'une compression mécanique est à présent évoqué comme le résultat d'une perception techniquement modifiée qui anéantit une vision du monde statique et anthropocentrique. Les soldats qui, pendant la nuit, font irruption dans le compartiment pour en sortir tout aussi subitement, sont perçus comme des apparitions spectrales : apparaissant et disparaissant avec la même fugacité que le paysage aperçu par la fenêtre du train, ils semblent " enfantés par la nuit et la guerre " et en ce sens "vaguement mythiques et fabuleux » (p. 1130,1133). Ainsi le véhicule moderne laisse-t-il place à une imagination mythique qui fait remonter la guerre industrialisée à la violence archaïque. Enfin, de même que le voyage en train stimule la perception auditive et visuelle, il stimule la mémoire. Depuis son compartiment, le passager peut voir son passé "dans une perspective télescopique ", se souvenant d'autres périples effectués en train à travers le monde violent de la fin des années 1930 (p. 1109). C'est bien par ce moyen qu'il est en mesure de s'imaginer l'Europe comme un " continent couturé de cicatrices ", constamment déchiré par les combats. Le train fait de lui une pièce de cette machine de guerre moderne toute rationnelle, mais ce faisant, il lui permet aussi d'en figurer les origines irrationnelles.

Mais plus encore, le train stimule son activité poétique. Dès le début du trajet, le protagoniste fait preuve d'une conscience aiguë du pouvoir suggestif du langage. Peu après le départ, son regard est attiré au sol par un journal dont il ne peut lire le titre que "machinalement " en raison des secousses régulières, ce qui signifie qu'il accorde moins d'attention à la signification du message qu'aux caractères imprimés eux-mêmes (p. 1106-1107). De ce fait, les lettres du titre cessent d'être des symboles purement conventionnels pour devenir des icônes évocatrices du déclenchement annoncé de la guerre, comme les "lettres de deuil » d'un faire-part (p. 1157) ou des « caractères monumentaux " gravés sur une tombe ${ }^{18}$ (p. 1163). Le même phénomène se produit avec le nom de la gare où le voyageur voit les gardes mobiles disparaître :

17. Voir W. Schivelbusch, op. cit., chap. 4. Cet effet particulier avait déjà été relevé en 1837 par Victor Hugo, aux yeux duquel des fleurs aperçues depuis un train en marche apparaissaient comme " des taches ou plutôt des raies rouges ou blanches ", tandis que le cheminot près de la voie ferrée semblait un "spectre debout [qui] parait et disparaît comme l'éclair" (Euvres complètes, Laffont, 1987, vol. 13, p. 611).

18. Pour la distinction sémantique entre signes symboliques et iconiques originellement établie par Peirce, voir A. W. Burks, "Icon, index and symbol ", Philosophy and Phenomenological Research, vol. 9, 19481949, p. 673-689. 
CULMONT-CHALINDREY, le nom surgissant soudain de la nuit, passant rapidement devant les yeux et de nouveau englouti, comme si, de même que les gardes mobiles, il avait été enfanté, fabriqué tout exprès, par les ténèbres, vaguement menaçant, avec ses lourdes consonances d'enclume et de chuintement de vapeur, pour se trouver là, loin de tout (de la lumière, des mers, des régions habitées) au fond de ce temps sans dimensions où le train continuait à rouler. (p. 1132)

Aperçu depuis le train, surgissant subitement comme les soldats fantomatiques, ce nom de lieu apparaît comme un signe iconique dont le signifiant évoque le bruit martial d'une enclume ("Culmont »/« enclume ») et la machine à vapeur («Chalindrey »/« chuintement »). En lisant le titre « machinalement », tandis que le train traverse le pays, le passage procède à une remotivation cratylienne de ce nom aux consonances étranges. En cela, il semble suivre l'exemple du jeune protagoniste $\mathrm{d}$ '̀̀ la recherche du temps perdu de Proust, qui pendant son voyage en train pour Balbec, passe de même "d'un nom à un autre nom ", interprétant celui de chaque gare comme l'image verbale de la localité qu'elle désigne ${ }^{19}$. La logique de cette remotivation est toutefois différente. Tandis que chez Proust les noms de gare évoquent des lieux individualisés et désirables, chez Simon, le "nom surgissant soudain dans la nuit " suggère le bruit de la machine de guerre qui anéantit toute singularité en donnant aux lieux des noms qui se ressemblent au point de se confondre ${ }^{20}$. En mettant ainsi au premier plan le potentiel évocatoire des mots, le voyage du protagoniste vers la guerre est aussi un voyage en écriture. De fait, l'éloignement brutal de son " univers normal " dans le Midi (p. 1134) et son transport dans le « cordon ombilical » du train (p. 1157) mènent finalement à une initiation littéraire qui fait de lui un romancier ${ }^{21}$. Comme il le rappelle durant le trajet, ses précédentes tentatives pour écrire un roman étaient vouées à l'échec; ses premiers efforts littéraires étaient tout aussi académiques que ses premières peintures sous l'influence du cubisme et de sa tendance à transformer tous les objets en formes géométriques, " en assemblages de tubes d'acier, de cônes et de sphères " (p. 1113, 1120). De toute évidence,

19. M. Proust, À la recherche du temps perdu, Gallimard, coll. «La Pléiade », 1987-1989, t. 2, p. 5. Sur le " cratylisme secondaire » de Proust, voir G. Genette, Mimologiques. Voyage en Cratylie, Le Seuil, 1976, p. 315-328.

20. Lors de la description du tracteur dans La Bataille de Pharsale (p. 661), le nom de la marque MAC coRMick fait l'objet d'un commentaire cratylien similaire. Pour de plus amples développements, voir notre étude Sprache und Gewalt bei Claude Simon. Interpretationen zu seinem Romanwerk der sechziger Jahre, Tübingen, Narr, 1992, p. 220-223.

21. Pour une analyse détaillée de ce processus initiatique, voir A. Duncan, Claude Simon: Adventures in words, Manchester, Manchester University Press, 1994, p. 128-152. 
cette autocritique rétrospective opère aussi comme une attaque contre l'absence de tout esprit critique de la part de la première avant-garde à l'égard de la technologie et de son enthousiasme naïf pour l'industrie moderne, un enthousiasme partagé par la " philosophie de la matière " marxiste qu'il étudiait à la même époque (p. 1115). Ce n'est qu'à son retour du camp de prisonniers qu'il réussit à écrire, stimulé par l'acacia de son jardin éclairé par une lumière électrique. Il semble désormais plutôt guidé par Proust et la seconde avant-garde surréaliste, qui présentent tous deux une image plus sombre de la machine, en soulignant à la fois son pouvoir destructeur et sa force productive dans le domaine de l'art. On peut aussi percevoir cette dimension dans la métaphore mythologique qui compare le train à un réseau de "cicatrices » et à la trace omniprésente d'une gigantesque corrida, image manifestement inspirée du Miroir de la tauromachie de Leiris, où l'auteur voit dans ce rituel sanglant un modèle pour une anthropologie esthétique centrée sur la violence ${ }^{22}$. C'est pourquoi le convoi militaire de L'Acacia ne perd pas ses connotations les plus sombres quand il se transforme en véhicule de la création littéraire : même après avoir ramené le passager chez lui à sa table de travail, il continue d'inspirer sa terreur originelle.

\section{LE CINÉMA DANS LES GÉORGIQUES}

L'intérêt de Simon pour la technologie est indissociable de celui qu'il éprouve pour les moyens de communication visuels. Parmi ceux-ci, la photographie occupe une place centrale et importante au sein de l'œuvre. Simon fait souvent référence à des clichés, qu'il s'agisse de leur production ou de leur réception ${ }^{23}$. Et plus souvent encore, il fait référence à la photographie comme modèle de perception et de remémoration, soulignant son affinité avec l'expérience du choc et du traumatisme, une affinité déjà relevée par Proust $^{24}$. Un certain nombre de ces références révèlent toutefois un intérêt particulier pour l'image en mouvement (c'est-à-dire l'image qui transgresse les limites techniques de la photographie), en se focalisant ou bien sur la

22. M. Leiris, Miroir de la tauromachie, Fata Morgana, 1984 [1938]. À propos de l'influence sur Simon de cet essai programmatique, voir notre article " Une poétique de la dépense. Claude Simon et le Collège de Sociologie ", Cahiers Claude Simon, n 4, 2008, p. 33-52.

23. Concernant l'œuvre photographique de Simon, voir Album d'un amateur, Remagen, Rommerskirchen, 1998; Photographies 1937-1970, Maeght, 1992; "Werkbuch ", Du, n 691, 1999, p. 28-73.

24. Pour une analyse plus approfondie, voir I. Albers, Claude Simon: moments photographiques, trad. L. Cassagnau, PU du Septentrion, 2007, p. 51-134. 
" trace fuligineuse " d'un cliché flou (Hist., p. 324) ou bien sur des technologies auxiliaires comme la lanterne magique $(A, \mathrm{p} .1206)$ ou le chronophoto$\operatorname{graphe}^{25}(J P$, p. 921). Il n'est dès lors pas surprenant de trouver chez Simon de nombreuses références au cinéma, et plus particulièrement quand cet art en est à ses débuts. Dans les romans des années 1950 et 1960, la référence est généralement d'ordre métaphorique. Ce n'est qu'avec Triptyque que le cinéma intègre la diégèse, avec divers films de sexe et d'action que l'on peut voir dans des conditions de projection plutôt atypiques et vieillottes, plus proches du cirque ou d'une cabine de peep-show que d'une salle de cinéma moderne ${ }^{26}$. Une autre expérience cinématographique tout aussi primitive est évoquée dans Les Géorgiques. Dans ce roman, le protagoniste, descendant et biographe du général révolutionnaire L.S.M., se souvient des dimanches après-midi qu'il passait au cinéma quand il était écolier dans une petite ville du Midi, à regarder des films muets depuis les mêmes places bon marché que les Gitans de la ville. Au fil de ses réminiscences, il livre une vision critique du cinéma, mettant surtout en avant la séduction agressive de ce média. À bien des égards, cette position correspond à celle de Walter Benjamin, qui, soulignant l'impact tactile du cinéma et la dimension rituelle de sa réception, met en garde contre le risque d'instrumentalisation politique de ces caractéristiques ${ }^{27}$. Chez Simon, la composante tactile est soulignée dès le début de la description. Avant que ne commence la projection du film, le public est assailli de publicités agressives, d'abord avec le "tintement [...] harcelant " de la sonnerie annonçant la séance, puis par l' "agressif rideau de réclames " qui recouvre l'écran (p. 777-779). Cette violente agression des sens se poursuit et augmente quand commence la projection du film. "[T]errifiante et vertigineuse ", la discontinuité des images cinématiques due au "flou sautillant de la mauvaise projection " heurte le spectateur; l'actrice qui apparaît à l'écran elle-même semble faire l'objet d'une tentative de viol, " comme si le projecteur de la cabine l'avait plaquée contre le $\operatorname{mur}^{28} »$ (p. 782). Les fré-

25. Voir L. Fraisse, "La lentille convexe de Claude Simon ", Poétique, nº 117, 1999, p. 40-41.

26. L'une des deux projections évoquées se déroule dans un cinéma délabré de banlieue, l'autre dans une grange dont le mur est recouvert d'affiches de cirque et perforé d'un trou pour épier à l'intérieur. Le trou est de temps à autre utilisé par deux garçons qui, à d'autres moments, examinent des morceaux de pellicule déchirée à l'aide d'une loupe. Les affiches de cirque évoquent le cinéma de Méliès; quant aux morceaux de pellicule, il rappellent le kinétoscope d'Edison qui n'admet qu'un seul spectateur et favorise en cela le voyeurisme.

27. W. Benjamin, L'Euvre d'art à l'époque de sa reproductibilité technique [1936], trad. L. Duroy, Allia, 2003.

28. Triptyque passe en revue de tels effets de manière exhaustive. Le film y est constamment associé à la rupture, à la destruction et à la violence. La première projection suscite des chocs visuels continus 
quentes interruptions de la projection ne permettent pas même au spectateur de reprendre son souffle; quand il regarde le sol, il peut voir les déchets jetés là par les autres spectateurs, "révélés soudain en gros plan " (p. 779). Bref, le cinéma ne fait que le confronter à un monde matériel agressif; avant, pendant et après la séance, il subit les mêmes " chocs brutaux ", le même "sentiment d'agression " qu'il endure pendant son voyage en train, quand, à travers la "lunette » des W.-C., il voit défiler sous ses yeux les détritus qui jonchent le ballast (p. 694). En outre, la description insiste également sur la dimension rituelle propre à toute séance de cinéma. Le public semble assister à " la célébration de quelque culte barbare " dont le centre serait l'écran qui est "le lieu d'une mystérieuse et lugubre cérémonie " (p. 777-779). Cette impression est rendue plus vive par la présence des Gitans qui occupent les «places dites "populaires" ", dans la mesure où ils font penser à une "horde barbare " constituée de "tribus primitives " (p. 782). De même que le train dans L'Acacia, le cinéma dans Les Géorgiques fait régresser une foule de la civilisation à la barbarie. Renforçant cette impression, la séquence est interrompue par l'évocation d'un camp de prisonniers allemand où une foule similaire s'assemble; dans ce passage, les projecteurs des miradors manifestent un semblable processus de régression, répétant ainsi l'effet du projecteur de film situé derrière les places populaires (p. 780-781). Cette analogie audacieuse a peutêtre été inspirée par Sartre qui, dans Les Mots, compare également les publics du cinéma de son enfance aux détenus d'un camp de prisonniers, en ce que les uns et les autres constituent une forme élémentaire de communauté ${ }^{29}$. Toutefois, qu'il s'agisse ou non d'une référence intertextuelle, l'analogie est clairement polémique. Sartre fait l'éloge du cinéma en lequel il perçoit l'absence de tout cérémonial, une libre "présence sans recul de chacun à tous "; Simon montre au contraire que la projection d'un film est indissociable d'un cérémonial quasi-barbare ${ }^{30}$.

Pour autant, il est encore un autre aspect que révèlent ces expériences remémorées de cinéma. Agressif, ce média est également présenté comme transgressif; pour reprendre les termes de Benjamin, il installe une distance

quand la pellicule se casse ( $T$, p. 772, 804), se coince (p. 821) ou brûle (p. 864), tandis qu'au même moment, la bande-son est interrompue (p. 760) ou déformée (p. 761, 775) par les hauts-parleurs et subit de ce fait la "violence du son " (p. 828).

29. J.-P. Sartre, Les Mots [1964], Gallimard, 1972, p. 104-105.

30. Sur l'attitude critique de Simon à l'égard de Sartre, voir V. Szöllösi-Brenig, Die "Ermordung " des Existentialismus oder das letzte Engagement. Künstlerische Selbstfindung im Frühwerk von Claude Simon zwischen Sartre und Merleau-Ponty, Tübingen, Narr, 1995, en particulier p. 121-132. 
salutaire entre le spectateur et son environnement familier ${ }^{31}$. D'un côté, les films qu'il voit lui offrent le poignant modèle d'une nouvelle vision de l'histoire, une vision qui n'est pas déterminée par les vieilles conventions narratives et qui s'oppose de ce fait à celle qu'il critique chez l'historien de la guerre civile O., alias George Orwell. Son expérience du présent se caractérise par des perceptions discontinues qui se manifestent particulièrement dans les effets de montage ou de trucage d'un film. Quand il voit un homme s'extraire brusquement de sous un tracteur à la ferme de L.S.M., il a l'impression d'" un de ces trucages de cinéma ou de music-hall, quand une scène se peuple, s'anime tout à coup, comme par magie " (p. 743); plus tard, dans l'appartement vide en compagnie d'un agent immobilier, il se sent à nouveau " comme au cinéma ", se rappelant ces effets spéciaux inventés par Méliès (p. 799). Ses propres souvenirs revêtent aussi la discontinuité d'un film. Quand, juste après l'évocation des séances de cinéma, il en vient à se rappeler un épisode de la guerre, il compare la transition entre les deux situations à un film muet où deux scènes éloignées dans le temps se trouvent directement juxtaposées, seulement séparées " par la brève apparition d'un placard» (p. 784). Quand il se figure comment $\mathrm{O}$. a pu réellement se rappeler la guerre, réalité qu'il a si bien dissimulée dans sa chronique de guerre conventionnelle, le narrateur évoque l'image d'un train en marche dans un effet proto-cinématographique :

c'était comme s'il se mouvait, engagé ou plutôt aspiré dans une sorte de tunnel, continuant machinalement à agiter ses jambes sous lui, rattrapé, dépassé par l'assourdissant ouragan d'un train express, rejeté par le souffle sur le côté, les lumières rapides des wagons l'extirpant de nouveau, le révélant chaque fois dans l'une des positions successives d'un homme en train de courir, comme dans ces kaléidoscopes où l'on peut voir les petites images fragmentaires d'un athlète immobilisé selon les attitudes décomposées de la course à pied, avec cette différence que le cylindre tournerait à l'envers, de sorte qu'il semblait pour ainsi dire avancer à reculons (p. 882)

Le moi remémoré est fragmenté en séries discontinues d'images instantanées, comme on peut les observer dans le cylindre tournant d'un kaléidoscope, ou plutôt d'un praxinoscope, l'un de ces précurseurs du cinéma au XIX ${ }^{\mathrm{e}}$ siècle; cette fragmentation est liée à l'effet quasi-stroboscopique d'un processus historique violent, suggéré une fois de plus par l'image d'un train qui passe dans un vacarme assourdissant. Enfin, le cinéma permet aussi de rendre compte du regard porté sur l'histoire par le narrateur dans une perspective collective. Travaillant à retracer les brusques revirements de fortune de son ancêtre à

31. W. Benjamin, «Petite histoire de la photographie » [1931], trad. A. Gunthert, Études photographiques, $\mathrm{n}^{\circ} 1,1996$. 
l'époque de la Révolution, il se souvient d'un vieux film burlesque, « un de ces films projetés à l'accéléré, avec ses foules, ses personnages ataxiques, aux gestes incohérents, inachevés» (p. 898). Ici, la discontinuité propre aux premiers films prend une dimension métahistorique, refusant une conception téléologique de l'histoire. Certes, Simon a déjà utilisé la plupart de ces métaphores cinématographiques dans ses romans précédents ${ }^{32}$. Mais dans Les Géorgiques pour la première fois, elles sont reliées métonymiquement à la révélation ${ }^{33}$ d'une expérience cinématographique concrète.

Cette expérience est également de l'ordre de la dépense extatique. Tandis que la discontinuité agressive du film transgresse les conventions de perception et de rétrospection, la ritualité régressive de sa réception transgresse les tabous culturels. Le " culte barbare " célébré par les occupants des "places populaires " peut bien être exploité à des fins commerciales ou politiques, il n'empêche qu'il donne lieu à une rencontre avec le domaine du sacrifice de soi, violent ou sexuel, jadis sacré, mais qui par la suite a fait l'objet d'une répression, avec ce que Leiris nomme «le sacré dans la vie quotidienne ${ }^{34}$ ». Issu d'un milieu aisé, l'écolier est irrésistiblement attiré par les places inconfortables du cinéma, précisément parce qu'elles représentent un " univers interdit " (p. 777). La dimension sexuelle de ce monde interdit est soulignée dès qu'il entre, ou plutôt " pénètre " dans la salle sombre (p. 778); implicite, l'image sexuelle devient explicite avec l'évocation de l'écran, " rectangle magique, virginal et impollué » (p. 779) et la comparaison de la fumée éclairée par le rayon du projecteur à un "placenta " flottant dans l'air (p. 782). En même temps, tout ce qui a trait à cet univers est investi d'une aura sacrée : le contrôleur des tickets apparaît comme un "personnage aux occultes pouvoirs ", et même les personnages figurés sur le rideau publicitaire sont promus " au rôle de tout-puissants gardiens d'un inépuisable trésor» (p. 777-779). Les Gitans font l'objet d'une semblable promotion. Ils incarnent la violence au royaume de l'interdit : dans la mesure où ils semblent détruire tout ce qu'ils touchent, y compris eux-mêmes, ils apparaissent comme l'incarnation humaine de « la violence à l'état pur» (p. 784). Et pourtant, c'est aussi exactement ce qui

32. Dans ces romans, le cinéma est aussi utilisé comme modèle perceptif ( $V$, p. 34; Hist., p. 169), remémoratif ( $C R$, p. 47; P, p. 422) ou historique (Herbe, p. 39).

33. «Eye-opener " (littéralement « qui ouvre les yeux ») dans le texte original (NdT).

34. M. Leiris, "Le sacré dans la vie quotidienne " [1938], dans D. Hollier (éd.), Le Collège de sociologie, Gallimard, $2^{e}$ éd., 1995, p. 94-119; pour plus de détails, voir notre article "Corps souillés : matérialité transgressive dans Histoire de Claude Simon » [1995], dans I. Albers et W. Nitsh (dir.), Lectures allemandes de Claude Simon, PU du Septentrion, 2013, p. 171-185. 
leur confère un halo de "divinités " archaïques et les rend encore plus fascinants que les vedettes sur l'écran (p. 782-783). Ainsi le cinéma constitue-t-il un espace sacré non pas en dépit, mais précisément en raison du cérémonial primitif qu'il abrite. Dans cette perspective, il marque un net contraste avec le théâtre ou l'église, ces sanctuaires officiels évoqués plus loin dans le roman. Ces deux espaces toutefois, considérés à la lumière de l'expérience du cinéma, s'avèrent être également des lieux barbares. Quand, pour la première fois de sa vie, le jeune spectateur assiste à une représentation théâtrale ou à la messe dominicale, il découvre de nouveaux « rituels à la fois sacrés et barbares " (p. 790) qui sont curieusement interdits à ceux avec qui il partage les dimanches après-midi. Ainsi, dans son expérience personnelle comme dans ses souvenirs, le cinéma vient toujours en premier, jamais en dernier; le rituel particulier qui lui est associé lui sert de guide vers d'autres rituels plus traditionnels et prestigieux, mais en fin de compte non moins barbares.

Bien sûr, ce rôle particulier du cinéma est lui-même produit par un usage audacieux des métaphores. Le personnage principal ne pourrait pas évoquer le cinéma comme une initiation moderne sans sa propre initiation à l'écriture qui s'accomplit loin des places populaires et qui est liée à la découverte des papiers du général ${ }^{35}$. Pourtant cette dernière étape, et peut-être la plus décisive dans son parcours épuisant à travers l'histoire, on peut considérer qu'elle s'origine dans cette rencontre avec ce nouveau média. Ce n'est qu'après que le cinéma lui a ouvert les yeux sur la violence cachée de sa propre sphère culturelle qu'il décide d'interroger son oncle Charles sur l'histoire violente de son ancêtre (p. 793). Ce lien narratif est souligné par un autre lien, rhétorique, qui résulte de la double signification du mot " placard ». La référence, dans l'évocation du film, à la "brève apparition d'un placard » (p. 784) convoque par association un autre "placard " situé dans la maison de famille : celui, enchâssé dans un mur, où étaient conservées les archives de l'ancêtre (p. 769770). Ainsi le roman indique-t-il un double point de départ à l'écriture. En mettant en avant les origines liées aux archives familiales, il s'inscrit en même temps dans le contexte du cinéma, comme un insert textuel qui apparaîtrait au milieu d'un film.

Ainsi, Les Géorgiques, roman souvent considéré comme un sommet dans l'œuvre de Simon et comme une clé pour en appréhender les principaux enjeux, se singularise aussi par une étroite interaction entre technologie et

35. Voir T. Klinkert, Bewahren und Löschen. Zur Proust-Rezeption bei Samuel Beckett, Claude Simon und Thomas Bernhard, Tübingen, Narr, 1996, p. 169-242. 
écriture. Si l'on veut porter un regard rétrospectif sur les romans antérieurs, il serait dès lors éclairant de choisir une perspective médiologique, en les étudiant non pas seulement comme des chambres aux miroirs autoréférentielles, comme des chambres d'écho intertextuelles ou comme des galeries d'art virtuelles, mais aussi comme des artefacts scripturaires hantés par ceux de l'ère technique qui lui font concurrence. Envisagés dans une telle perspective, on pourrait peut-être montrer que les personnages et protagonistes simoniens ont presque autant appris dans les trains et les cinémas que dans les musées et les bibliothèques.

Article originellement publié sous le titre "Supplementary Organs : Media and Machinery in the Late Novels of Claude Simon", dans J. H. Duffy et A. B. Duncan (dir.), Claude Simon. A Retrospective, Liverpool, Liverpool University Press, 2002, p. 152-167. Trad. David Zemmour, revue par l'auteur. 